\title{
O DISCURSO DE PESSOAS ACOMETIDAS POR TUBERCULOSE SOBRE A ADESÃO AO TRATAMENTO ${ }^{1}$
}

\author{
THE DISCOURSE OF PEOPLE TAKEN ILL BY TUBERCULOSIS \\ ABOUT THE TREATMENT ADHESION
}

\section{EL DISCURSO DE PERSONAS AFECTADAS POR TUBERCULOSIS SOBRE LA ADHESIÓN AL TRATAMIENTO}

\author{
Débora César de Souza Rodrigues* \\ Annelissa Andrade Virgínio de Oliveira ** \\ SÉFora Luana Evangelista de Andrade ${ }^{* * *}$ \\ Edna Marília Nóbrega Fonseca de Araújo ${ }^{* * *}$ \\ Ana Maria Cavalcante Lopes ${ }^{* * * *}$ \\ LENILDE DUARTE DE SÁ ${ }^{* * * * *}$
}

\begin{abstract}
RESUMO
A tuberculose é uma doença infectocontagiosa que permanece com altos índices de adoecimento em vários países e a não adesão ao tratamento tem reduzido os índices de cura. Objetivo: Identificar, mediante o discurso de pessoas acometidas pela doença, fatores e significados que favorecem a adesão ao tratamento específico. Método: Para a coleta dos dados foi empregada a entrevista em profundidade. A análise foi fundamentada no dispositivo teórico-analítico da Análise de Discurso de linha Francesa. Resultados: A forma como profissionais acolhem os doentes, principalmente o enfermeiro, bem como a o apoio dos familiares, são fatores decisivos para a adesão ao tratamento. Sobre a posição do sujeito em relação ao cuidado, observa-se que se filia a concepção de integralidade quando atribuída a organização das práticas de saúde. Conclusão: Neste aspecto, ressalta-se a importância das singularidades das pessoas adoecidas por tuberculose na elaboração dos projetos terapêuticos.
\end{abstract}

Palavras chave: Tuberculose, adesão à medicação, atenção primária à saúde, enfermagem em saúde comunitária.

\footnotetext{
${ }^{1}$ Suporte financeiro Projecto: Fatores associados à adesão ao tratamento da tuberculose, aprovado e financiado pelo programa IICOHRTA-AIDS/TB Nº 1 V2RTW006883-06 através da parceria com CRPHF/ENSP/FIOCRUZ Fundação para o Desenvolvimento Científico e Tecnológico em Saúde No ENSP-011-LIV-10-2-2 em 2011.

* Enfermeira da Estratégia de Saúde da Família, Mestre em Enfermagem pela Universidade Federal da Paraíba/UFPB, pesquisadora do Grupo de Estudos e Qualificação em Tuberculose da Paraíba TB/PB. João Pessoa- Paraíba, Brasil. Email: deboracesarrufpb@gmail.com. Autor correspondente.

** Enfermeira Assistencial do Hospital Universitário Lauro Wanderley/HULW, Doutora em Saúde Coletiva pela Universidade Federal da Paraíba/UFPB, pesquisadora do Grupo de Estudos e Qualificação em Tuberculose da Paraíba TB/PB. João Pessoa- Paraíba, Brasil. Email: annelissa.andrade@gmail.com.

${ }^{* * *}$ Enfermeira. Docente na Faculdade Uninassau. Doutoranda no Programa de Pós Graduação em Enfermagem da Universidade Federal da Paraíba/UFPB; pesquisadora do Grupo de Estudos e Qualificação em Tuberculose da Paraíba TB/PB. João Pessoa-Paraíba, Brasil. Email: seforaejoab@hotmail.com.

**** Enfermeira residente na ênfase Saúde do Idoso no Hospital Universitário Lauro Wanderley/HULW; pesquisadora do Grupo de Estudos e Qualificação em Tuberculose da Paraíba TB/PB. João Pessoa- Paraíba, Brasil. Email: edna_marilia@ hotmail.com.

${ }_{* * * * *}$ Enfermeira. Docente da Universidade Federal da Paraíba/UFPB, Doutora pelo Programa de Pós- Graduação em Enfermagem da Universidade Federal da Paraíba/UFPB; pesquisadora do Grupo de Estudos e Qualificação em Tuberculose da Paraíba TB/PB. João Pessoa- Paraíba, Brasil. Email: anamariacavalcantelopes@gmail.com.

${ }^{x * x+x+*}$ Enfermeira. Docente da Universidade Federal da Paraíba/UFPB. Pós-Doutora pela Escola de Enfermagem da Universidade de São Paulo; pesquisadora do Grupo de Estudos e Qualificação em Tuberculose da Paraíba TB/PB. João Pessoa- Paraíba, Brasil. Email: sa.lenilde@gmail.com.
} 


\begin{abstract}
Tuberculosis is an infectious contagious disease that remains at a high illness rate in several countries and the non-adhesion to the treatment has reduced the healing rate. Objective: The study aimed at identifying, through the discourse of people infected by tuberculosis, factors and meanings that benefit the adhesion to the specific treatment. Method: For the data collection an in-depth interview was applied. The analysis was based on the theoretical-analytical framework of the French school of Discourse Analysis. Results: The results revealed that the manner in which professionals welcome the patient, mainly the nurse, as well as the relatives' support, are decisive factors to the adhesion of the treatment. Concerning the subject's stand regarding the care, one observes that it is linked to the concept of integrality as attributed to the organization of health practices. Conclusion: In this aspect, the importance of the singularities of the people infected by tuberculosis stands out in the elaboration of the therapeutic projects.
\end{abstract}

Key words: Tuberculosis, medication adherence, health primary care, community health nursing.

\title{
RESUMEN
}

La tuberculosis es una enfermedad infectocontagiosa con altos índices de enfermos en varios países y la no adhesión al tratamiento ha reducido los índices de cura. Objetivo: Identificar, mediante el discurso de personas afectadas por la enfermedad, factores y significados que favorecen la adhesión al tratamiento específico. Método: Para la recolección de datos fue empleada la entrevista en profundidad. El análisis fue fundamentado en el dispositivo teórico-analítico del Análisis de Discurso de línea Francesa. Resultados: La forma como los profesionales acogen a los enfermos, principalmente el enfermero, así como el apoyo de los familiares, son factores decisivos para la adhesión al tratamiento. Sobre la posición del sujeto en relación al cuidado, se observa que se mantiene la concepción de integralidad cuando es atribuida a la organización de las prácticas de salud. Conclusión: En este aspecto se resalta la importancia de las singularidades de las personas afectadas por tuberculosis en la elaboración de los proyectos terapéuticos.

Palabras clave: Tuberculosis, adhesión a la medicación, atención primaria a la salud, enfermería en salud comunitaria.

Fecha recepción: 12/05/15 Fecha aceptación: 21/01/17

\section{INTRODUÇÃO}

A Tuberculose (TB) é considerada uma das doenças infectocontagiosas com mais altos índices de adoecimento em vários países e destaca-se como aquela que mais mata no mundo. Esta doença, a qual muitos associam ao passado, encontra-se atualmente em franca expansão, atingindo principalmente os segmentos mais pobres, os vulneráveis, os marginalizados (1).

$\mathrm{O}$ controle da TB permanece como um sério desafio, embora a mesma seja uma doença curável. Nesse sentido, as dificuldades para o seu controle voltam-se aos constituintes organizacionais dos serviços de saúde e do próprio comportamento humano (2), incluindo, neste aspecto, doentes e profissionais de saúde.

Reconhece-se que nos países em desenvolvimento, além dos desafios relacionados às posturas das pessoas adoecidas, as desigualdades e carências sociais, o aumento da pobreza, o crescimento desordenado das cidades e da população associados à falta de acesso aos serviços de saúde, dificultam o controle e o manejo da tuberculose, assim 
como a adesão ao seu tratamento $(3,4)$.

$\mathrm{Na}$ tentativa de explicar a baixa adesão ao tratamento, evidenciada por altos índices de abandono, diversos fatores tem sido relatados, tais como: uso de álcool; interrupção de benefícios, tratamento autoadministrado, falta de suporte familiar, fragilidade no vínculo entre doente e profissional e ausência de trabalho em equipe nos serviços de saúde (5, 6). Concorrem ainda para o aumento deste índice, tornando cada vez mais complexo o problema do abandono do tratamento da TB: o uso de drogas, as situações de vulnerabilidade social e a falta de acesso à informação (7).

É importante destacar que a TB é uma das doenças consideradas prioritárias na Política Nacional de Atenção Básica (PNAB) brasileira e, desde o ano de 2006, o Ministério da Saúde (MS) tem intensificado a descentralização das ações de diagnóstico, controle e tratamento da doença para os serviços da Atenção Básica $(A B)(8)$, pois é nesse nível de atenção que deve ser oferecido todo o cuidado ao doente de TB. Na AB a Estratégia de Saúde da Família (ESF) é estruturante dos sistemas municipais de saúde, por remodelar a assistência à saúde e promover uma dinamicidade nova na organização dos serviços e ações, neles incluídas as medidas para o controle da $\operatorname{TB}(9,10)$.

Apesar de significar um avanço, os desafios do processo de descentralização das ações de controle da TB são significativos, principalmente no que concerne à fragmentação de regimes de tratamento decorrendo em maiores dificuldades para realizar o Tratamento Diretamente Observado (TDO) $(11,12)$.

João Pessoa, capital do Estado da Paraíba, é considerado um município prioritário para o controle da doença pelo Programa Nacional de Controle da Tuberculose (PNCT). No ano de 2014 este municipio apresentou um coeficiente de incidência de TB por todas as formas de 35,7/100.000 habitantes, um coeficiente de mortalidade de 2,7/100.000 habitantes e percentual de abandono de 18,6\%
(13). Vale ressaltar que neste município a taxa de abandono é superior a recomendada pela OMS, o que realça o conceito-análise tratado neste artigo, ou seja, a adesão ao tratamento da TB.

Mesmo existindo dificuldades para manter o doente em tratamento, em Unidades de Saúde da Família (USF) do município de João Pessoa, tem se observado experiências exitosas, levando a pessoa acometida por TB a obter alta por cura, apesar de suas condições e comorbidades que concorrem para o abandono da terapêutica. Tal fato inquietou os presentes autores a buscar resposta ao seguinte questionamento: Que sentidos revelam os discursos de pessoas adoecidas por TB em relação aos fatores que favorecem a adesão do tratamento nas USF?

Uma vez que, pela a análise de discurso $(\mathrm{AD})$, procura-se compreender a língua fazendo sentido (14), este estudo teve como objetivo identificar, por meio do discurso de pessoas acometidas por TB e assistidas em USF, fatores e significados favorecedores da adesão ao tratamento.

\section{MÉTODO}

Pesquisa qualitativa que utiliza como referencial teórico-analítico a Análise de Discurso $(\mathrm{AD})$, dispositivo que concebe a linguagem como mediação necessária entre o homem e a realidade natural e social, tornando possível tanto a permanência e a continuidade quanto o deslocamento e a transformação do homem e da realidade na qual ele vive (14).

Contribuíram com o estudo 8 (oito) pessoas que foram acometidas por TB pulmonar e que iniciaram e concluíram o tratamento em USFs do município de João Pessoa, obtendo a cura como desfecho terapêutico. A identificação e localização dos sujeitos foram possíveis por meio dos dados do Sistema de Informação de Agravos de Notificação (SINAN). 
Os sujeitos foram caracterizados por pseudônimos que se referem a personagens da história e da ficção que foram acometidos pela TB. A escolha dos codinomes para cada sujeito foi baseada em alguma característica da vida de ambos que se assemelhassem. Os pseudônimos selecionados foram: Martins Fontes, um morador de rua solitário e etilista, entrevistado 1; Barbosa de Freitas, jovem casado, boêmio, etilista e fumante, entrevistado 2; Rainha Vitória da Suécia, mãe solteira, cuidou da filha Marguerite quando doente de TB, entrevistado 3; Marguerite (A dama das Camélias) jovem boêmia, criada apenas pela mãe, entrevistado 4; Noel Rosa. boêmio etilista e fumante, entrevistado 5; Katherine Mansfield, jovem estudante que conviveu com dificuldade de relacionamento afetivo com a mãe, entrevistado 6; Manoel Bandeira, boêmio, etilista e fumante, entrevistado 7; Modigliani, jovem boêmio, etilista e usuário de drogas ilícitas, entrevistado 8.

Para coleta das informações foi utilizada a técnica da entrevista em profundidade, a qual foi realizada nos meses de agosto e setembro de 2012, com o auxílio de um equipamento de gravação de áudio.

O estudo foi autorizado pela Secretaria Municipal de Saúde de João Pessoa e aprovado em 27 de julho de 2011 pelo Comitê de Ética em Pesquisa do Centro de Ciências da Saúde da Universidade Federal da Paraíba CCS/UFPB, conforme protocolo No 0113/2011, atendendo às orientações sobre as pesquisas que envolvem seres humanos contido na resolução 466/12 do Conselho Nacional de Saúde (CNS) (15).
A proposta metodológica do dispositivo da AD (16) propõe três perguntas heurísticas para o processo de análise do corpus discursivo. A primeira refere-se à definição do conceito-análise, a segunda diz respeito à formulação do sentido desse conceito-análise por meio da textualização e de suas marcas textuais ou linguísticas e a terceira e última pergunta busca relacionar o sentido construído com formações discursivas e ideológicas (16).

Nesse estudo, o conceito-análise definido foi "Adesão ao tratamento da Tuberculose", esse conceito se sustenta por meio das marcas textuais que se expressam no corpus discursivo construído a partir dos discursos do doente de TB.

A partir da verificação da recorrência das marcas textuais, agrupamento das mesmas e a construção de enunciados de base, emergiram duas formações discursivas (FD's): a primeira denominou-se "Cuidado dos profissionais e o vínculo estabelecido com o doente favorecem à adesão ao tratamento da TB" e a segunda "Cuidado dos familiares favorece à adesão ao tratamento da TB".

\section{RESULTADOS}

O quadro 1 contempla o agrupamento das marcas textuais recorrentes analisadas, o enunciado de base construído, bem como as duas formações discursivas que nortearão a discussão do conceito análise proposto. 
Quadro 1. Conceito-Análise: “Adesão ao tratamento da Tuberculose”.

\begin{tabular}{|c|c|c|}
\hline Marcas textuais & Enunciado de Base & Formação Discursiva \\
\hline $\begin{array}{l}\text { “(...) todo dia eu tinha que ir na casa de S. (ACS) } \\
\text { pegar os comprimidos... Eu era bem recebido... } \\
\text { tomava o remédio na casa dela, no copo dela (...)" } \\
\text { Martins Fontes. } \\
\text { "(...) o incentivo (...) da enfermeira, da médica } \\
\text { e também da Agente de saúde, me ajudou muito } \\
\text { a querer continuar até o fim, todas tinham um } \\
\text { cuidado especial comigo." Barbosa de Freitas. } \\
\text { "A enfermeira era sempre muito cuidadosa, aliás, } \\
\text { todos de lá são, mas ela quem me acompanhou, } \\
\text { a que fez tudo, quem pedia exames era ela." } \\
\text { Marguerite. } \\
\text { "(...) Quem ficou mais próximo de mim, foi a } \\
\text { enfermeira...ela que fazia o acompanhamento } \\
\text { dos remédios (...)" Manoel Bandeira. }\end{array}$ & $\begin{array}{l}\text { Atenção e cuidado dos } \\
\text { profissionais da equipe } \\
\text { durante o tratamento }\end{array}$ & $\begin{array}{c}\text { Cuidado dos } \\
\text { profissionais e o } \\
\text { vínculo estabelecido } \\
\text { com o doente } \\
\text { favorecem à adesão ao } \\
\text { tratamento da TB }\end{array}$ \\
\hline $\begin{array}{l}\text { “(...) Os exames acusaram essa doença... Mas, de } \\
\text { jeito nenhum minha família virou as costas para } \\
\text { mim graças a Deus, pelo contrário, me deram } \\
\text { muita força, me deram muito apoio, ficaram aqui } \\
\text { comigo (...)” Marguerite. } \\
\text { “(...) a minha filha cuidava de tudo, para eu ter } \\
\text { uma alimentação melhor, mais adequada, para } \\
\text { eu poder recuperar o peso perdido." Noel Rosa. } \\
\text { “(...) Foi a minha esposa... ela quem me } \\
\text { influenciou. Na verdade eu agradeço tudo a ela } \\
\text { por que... Foi por causa dela que eu fui fazer os } \\
\text { exames (...) minha avó nunca deixava faltar nada } \\
\text { não, para eu me alimentar bem, sempre deixava } \\
\text { alguma coisa pronta para mim (...)” Modigliani. }\end{array}$ & $\begin{array}{l}\text { Apoio e ajuda da } \\
\text { família durante o } \\
\text { tratamento }\end{array}$ & $\begin{array}{l}\text { Cuidado dos familiares } \\
\text { favorece à adesão ao } \\
\text { tratamento da TB }\end{array}$ \\
\hline
\end{tabular}

\section{DISCUSSÃO E CONCLUSÃO}

A utilização dos serviços representa a funcionalidade dos sistemas de saúde e o processo de utilização desses é resultante da interação do comportamento do indivíduo, que procura cuidados, e a do profissional que o conduz pelo interior do sistema. Os profissionais, em sua função, devem garantir a intensidade e o tipo de recursos consumidos para resolver os problemas de saúde das pessoas adoecidas (17).

Em se tratando da oferta de cuidado na ESF, os cenários, os sujeitos e as linguagens apresentam intenso potencial para recons- 
trução das práticas no âmbito da atenção à saúde. Para um cuidado fundamentado no princípio da integralidade, o usuário deve ser o protagonista, mas pressupondo a presença ativa do outro, neste caso do profissional de saúde $(18,19)$. A ESF tem a lógica de produção do cuidado e direciona-se, também, pelo cumprimento das dimensões do que se define como acesso e vínculo (20).

$\mathrm{Na}$ ESF o contato constante com o território, a integração de práticas preventivas, educativas e curativas mais próximas da vida cotidiana da população e, principalmente, dos grupos mais vulneráveis, pode vir a ampliar o acesso aos recursos diagnósticos/ terapêuticos e promover a construção de vínculos entre profissionais e pessoas com TB $(21,22)$. Na AB o cuidado prestado por profissionais que compõem as equipes de saúde faz com que sejam criados vínculos de co-responsabilidade, o que facilita a identificação dos problemas de saúde da comunidade e, consequentemente, o envidamento de respostas (23).

As marcas textuais que sustentam a primeira FD "Cuidado dos profissionais e o vínculo estabelecido com o doente favorecem à adesão ao tratamento da TB", explicitam esse atendimento fortalecido com o vínculo e o cuidado, sendo os elementos essenciais para a continuidade do tratamento da TB como pode ser observado na marca textual extraída do discurso de Martins Fontes: “(...) todo dia eu tinha que ir na casa de S. (ACS) pegar os comprimidos... Eu era bem recebido... tomava o remédio na casa dela, no copo dela(...)".

O profissional referenciado nesse cuidado (S.), tratava-se da Agente Comunitário de Saúde (ACS). É evidente que a atenção oferecida pela ACS no acompanhamento da tomada do medicamento de Martins Fontes influenciou sua adesão ao tratamento, o dito "tomava o remédio na casa dela, no copo dela" pode significar como as pessoas acometidas pela TB sofrem preconceito e são excluídos do simples convívio social e das atividades diárias como compartilhar utensílios, explicitando o quanto a atenção da ACS foi importante para promover o aceite do tratamento pelo sujeito.

Assim, observa-se que a inserção do ACS envolve um conceito que, sob as mais diferentes formas, nomenclaturas e racionalidades, aparece em várias partes do mundo, ou seja, a ideia essencial de elo entre a comunidade e o sistema de saúde. E, para tanto, a importância e necessidade de construção de vínculo. $\mathrm{O}$ vínculo permeia o trabalho do ACS e potencializa sua responsabilização com a saúde das famílias (24).

$\mathrm{Na}$ perspectiva do que foi dito por Martins Fontes a respeito do que ele necessitava, cuidar em saúde está associado à perspectiva de se prestar atenção ou dirigir intervenções a um indivíduo ou a um grupo de maneira a envolver um conjunto de procedimentos tecnicamente orientados para o bom êxito de um determinado tratamento (25). O não dito aponta o cuidado no sentido mais amplo, como sinônimo do termo desvelo, definido como o "cuidado e vigilância contínua", diligência, zelo e solicitude (25).

O profissional de saúde se posiciona frente ao outro no ato de cuidar, constituindose assim um grande desafio ao nosso entendimento o colocar-se em movimento na inter-relação de um encontro de faces (26).

Estudos comprovam que se deve atribuir como prioridade no processo assistencial, além da supervisão da medicação, a melhoria da relação entre o usuário e o trabalhador da saúde. Deve-se, portanto, ser estabelecido o vínculo, fator que traz aspectos importantes como valorização de queixas, subjetividades, comunicação social, relação de escuta e de empatia com o usuário (27).

As marcas textuais abaixo corroboram com a posição dos autores acima (27), revelando que um dos elementos importantes do referido serviço de saúde para o fortalecimento à adesão ao tratamento da TB, foi a atenção e o cuidado dos profissionais: 
“(...) o incentivo (...) da enfermeira, da médica e também da Agente de saúde, me ajudou muito a querer continuar até o fim, todas tinham um cuidado especial comigo." Barbosa de Freitas.

"A enfermeira era sempre muito cuidadosa, aliás, todos de lá são, mas ela quem me acompanhou, a que fez tudo, quem pedia exames era ela." Marguerite.

“(...) Quem ficou mais próximo de mim foi a enfermeira... ela que fazia o acompanhamento dos remédios (...)" Manoel Bandeira.

Para a AD o trabalho simbólico do discurso está na base da produção da existência humana (14). No cuidado referido nos discursos acima, evidencia-se, na perspectiva do simbólico, a importância do enfermeiro, uma vez que várias marcas textuais apontam este profissional como o que mais se aproxima e oferece atenção à pessoa adoecida por TB. Nas equipes da ESF geralmente é o enfermeiro quem acompanha o doente durante o tratamento. Vale ressaltar que o enfermeiro no Brasil está presente desde as primeiras ações de saúde pública voltadas para o controle da doença (28), conferindo-lhe especial destaque (29-30), o que sem dúvida o fortalece simbolicamente na história da saúde, como um agente vinculado ao cuidado do paciente acometido por TB.

Neste estudo o dito revela a participação do enfermeiro na adesão ao tratamento, confirmando assim, a sua importância no núcleo de profissionais que inclusos no processo de trabalho da Equipe de saúde da Família, destacam-se como protagonistas na história das ações de controle da TB e com o compromisso com o cuidado ao doente deste agravo (31), acentuando-o, assim, significativamente como um profissional fundamental para $o$ efetivo controle da doença.

Em relação ao vínculo, este pressupõe a existência de uma fonte regular de atenção, e seu uso ao longo do tempo requer o estabelecimento de fortes laços interpessoais que reflitam a cooperação mútua entre as pessoas da comunidade e os profissionais de saúde
(20). E, quando se trata do controle da TB, ou do fortalecimento da continuidade dos usuários ao tratamento, constitui-se como um dos principais elementos estruturantes, uma vez que permeia atributos como a responsabilização, a integralidade e a humanização (27).

Em relação à segunda FD, "Cuidado da família favorece à adesão ao tratamento da TB", é importante destacar que os benefícios concedidos pelas redes de apoio a usuários que fazem ou que precisam iniciar tratamentos medicamentosos em muitos casos garantem a adesão à terapêutica. As marcas textuais mais evidentes mostram que o apoio dos familiares fortaleceu esta etapa importante para obter a cura da doença.

“(...) Os exames acusaram essa doença... Mas, de jeito nenhum minha família virou as costas para mim graças a Deus, pelo contrário, me deram muita força, me deram muito apoio, ficaram aqui comigo(...)" Marguerite.

“(...) a minha filha cuidava de tudo, para eu ter uma alimentação melhor, mais adequada, para eu poder recuperar o peso perdido." Noel Rosa. “(...) Foi a minha esposa... ela quem me influenciou. Na verdade eu agradeço tudo a ela porque...Foi por causa dela que eu fui fazer os exames (...)minha avó nunca deixava faltar nada não, para eu me alimentar bem, sempre deixava alguma coisa pronta para mim (...)" Modigliani.

Nenhuma pessoa vive no mais completo isolamento, sem ser influenciada pelo meio em que vive e pelas pessoas que a rodeiam (32). A família é um sistema interligado e cada um de seus membros tem influência sobre o outro, sendo que o adoecimento de um dos integrantes tem reflexos no comportamento e no estado emocional e até biológico dos demais (33).

A análise das marcas textuais que compõe esta formação discursiva revelam os significados atribuídos em relação à importância da família na manutenção do tratamento 
pelo usuário, a família consegue identificar necessidades, sofrimentos, sentimentos de e apoio. O afeto familiar permite que o sujeito doente tenha estabilidade para lutar contra a doença, ajuda-o a conseguir suprir suas carências emocionais e a aceitar a situação de encontrar-se adoecido, contribuição na adesão e continuidade nos tratamentos (33). Neste sentido, durante o tratamento e a reabilitação do doente de TB, o apoio que os familiares, amigos ou redes de apoio prestam, é de fundamental importância, pois contribuirá de forma positiva na adesão ao tratamento, manutenção e obtenção da cura.

Sabe-se que a adesão ao tratamento da TB relaciona-se diretamente ao conhecimento do doente acerca de sua doença, da sua responsabilidade consigo, sua capacidade para autocuidado e o seu desejo de se curar. No entanto, além disso, o sucesso da adesão está ligado ao apoio da família (34).

Portanto, sendo perceptível que o apoio das redes, como a família, constitui fator que potencializa a adesão ao tratamento da TB, como mostram os discursos, os profissionais de saúde devem estar atentos e dispostos à inclusão/participação de familiares, tanto no cuidado ao usuário, quanto no planejamento e execução do plano de cuidados.

Sobre a posição dos sujeitos em relação à concepção de cuidado, esta se filia à perspectiva da integralidade, quando relacionada a práticas de saúde (35). Ou seja, quando os sentidos se referem aos encontros entre equipe de saúde e as pessoas que demandam cuidado, de modo que os projetos terapêuticos surjam do diálogo entre profissionais de saúde e os usuários que buscam os serviços de saúde. Ressalta-se que a capacidade de compreender o contexto específico dos diferentes encontros é a característica chave para a existência desse diálogo. Assim, nas práticas de cuidado, singularidades e subjetividades das pessoas adoecidas devem ser consideradas, de modo que os profissionais possam elaborar projetos terapêuticos embasados em seus conhecimentos e naqueles trazidos pelo ou- tro a partir de seus sofrimentos, de suas expectativas, de seus temores e de seus desejos (35).

Na presente pesquisa os discursos revelaram que a forma como a pessoa com tuberculose é cuidada por profissionais de saúde e familiares é determinante para a adesão e continuidade do tratamento. Neste sentido, destaca-se, em relação ao profissional, o vínculo estabelecido na relação de cuidado, a não demostração de estigma e/ou preconceito, que são interpretadas pelos sujeitos como atitudes de acolhimento. Na USF o enfermeiro sobressai-se como o profissional mais atuante no processo terapêutico.

No sucesso alcançado pelos sujeitos em aderirem e concluírem o tratamento, além dos profissionais de saúde, os discursos revelaram ser importante apoio da família. Interpreta-se que o afeto familiar colabore para que a pessoa doente se fortaleça para enfrentar a doença, aderir e manter-se firme em relação ao tratamento.

Conclui-se, portanto, que nos serviços da $\mathrm{AB}$, notadamente nas USF, a forma como os profissionais acolhem os doentes de $\mathrm{TB}$ -principalmente o enfermeiro- bem como o apoio dos familiares, são fatores decisivos para a conduta em relação ao tratamento. Quanto à concepção que interpela os sujeitos em relação ao cuidado, depreende-se que os discursos evocam sentidos relacionados à integralidade no que concerne a organização das práticas vinculadas ao cuidado em saúde.

Agradecimentos: International Implementation, Clinical, Operational and Health Services Research Training Award for AIDS and Tuberculosis (IICOHRTA-AIDS/TB).

\section{REFERÊNCIAS}

1. Maciel ELN. Estratégias da agenda pós2015 para o controle da tuberculose no Brasil: desafios e oportunidades. Epide- 
miol Serv Saude. 2016; 25(2): 423-426.

2. Brunello MEF, Cerqueira DF, Pinto IC, Arcênio RA, Gonzales IC, Villa TCS, et al. Vínculo doente-profissional de saúde na atenção a pacientes com tuberculose. Acta Paul Enferm. 2009; 22(2): 176-82.

3. Santos NSGM, Santos MLSG, Vendramini SHF, Villa TCS, Ruffino-Netto A, Chiaravalloti-Neto F, et al. Tuberculose e Análise Espacial: revisão da literatura. Cienc. enferm. 2014; XX (2): 117-129.

4. Cioran V, Mincă DG, Brîndușe LA. Costs-of-illness evaluation methodology due to tuberculosis in homeless adults in $\mathrm{Bu}-$ charest. Acta Medica Transilvanica. 2015; 20(2): 13-15.

5. Cruz MM, Cardoso GCP, Abreu DMF, Decotelli PV, Chrispim PP, Borenstein JS, et al. Adesão ao tratamento diretamente observado da tuberculose - o sentido atribuído pelos usuários e profissionais de saúde em duas regiões administrativas do município do Rio de Janeiro. Cad. Saude Colet. 2012; 20(2): 217-24.

6. Furlan MCR, Oliveira SP, Marcon, SS. Fatores associados ao abandono do tratamento de tuberculose no estado do Paraná. Acta Paul Enferm. 2012; 25(Spec 1): 108-114.

7. Alves RS, Souza KMJ, Oliveira AAV, Palha PF, Nogueira JA, Sá LD. Abandono do tratamento da tuberculose e integralidade da atenção na estratégia saúde da família. Texto contexto - enferm. 2012; 21(3): 650-657.

8. Ministério da Saúde Brasil. Secretaria de Atenção à Saúde. Departamento de Atenção Básica. Política Nacional de Atenção Básica. Brasília: Ministério da Saúde; 2012. $110 \mathrm{p}$.

9. Escorel S, Giovanella L, Mendonça HM, Senna MCM. O Programa de Saúde da Família e a construção de um novo modelo para a atenção básica no Brasil. Rev Panam Salud Publica. 2007; 21(2): 164176.

10. Silva LA, Casotti CA, Chaves SCL. A pro- dução científica brasileira sobre a Estratégia Saúde da Família e a mudança no modelo de atenção. Cien Saude Colet. 2013; 18(1): 221-232.

11. Frieden TR, Driver CR. Tuberculosis control: past 10 years and the future progress. Tuberculosis (Edinb). 2003; 83(1-3): 8285.

12. Cecilio HPM, Marcon SS. O tratamento diretamente observado da tuberculose na opinião de profissionais de saúde. Rev enferm UERJ. 2016; 24(1): e8425.

13. Ministério da Saúde Brasil. Secretaria de Vigilância em Saúde. Detectar, tratar e curar: desafios e estratégias brasileiras frente à tuberculose. Boletim Epidemiológico. 2015; 46(9).

14. Orlandi EP. Análise do discurso: princípios e procedimentos 8 ed. Campinas, SP: Pontes, 2009. 109 p.

15. Ministério da Saúde Brasil. Conselho Nacional de Saúde, Resolução $\mathrm{N}^{\circ} 466$, de 12 de dezembro de 2012: Diretrizes e Normas Regulamentadoras de Pesquisas envolvendo seres humanos. Brasília: Ministério da Saúde; 2012.

16. Freire SA. Análise de discurso: procedimentos metodológicos. Manaus: Instituto Census; 2014. 54 p.

17. Souza ECF, Vilar RLA, Dantas NSP, Uchoa AC, Rocha PM. Acesso e Acolhimento na Atenção Básica: uma análise da percepção dos usuários e profissionais de saúde. Cad. Saude Publica. 2008; 24 (Suppl 1): S100-S110.

18. Ayres JRCM. Cuidado e reconstrução das práticas de saúde. En: Minayo MCS, Coimbra Jr CEA, orgs. Críticas e atuantes: Ciências Sociais e Humanas em saúde na América Latina. Rio de Janeiro: Editora Fiocruz; 2005, p. 91-108.

19. Kalichman AO, Ayres JRCM. Integralidade e tecnologias de atenção à saúde: uma narrativa sobre contribuições conceituais à construção do princípio da integralidade no SUS. Cad Saude Publica. 2016; 32(8): e00183415. 
20. Starfield B. Atenção primária: equilíbrio entre necessidades de saúde, serviços e tecnologia. Brasília: UNESCO, Ministério da Saúde Brasil; 2002. 726 p.

21. Marcolino ABL, Nogueira JA, RuffinoNetto A, Moraes RM, Sá LD, Scatena TC. Avaliação do acesso às ações de controle da tuberculose no contexto das equipes de saúde da família de Bayeux - PB. Rev Bras Epidemiol. 2009; 12(2): 144-57.

22. Sá LD, Gomes AL, Nogueira JA, Villa TC, de Souza KM, Palha PF. Intersectorality and bonding in tuberculosis control in family health. Rev Lat Am Enfermagem. 2011; 19(2): 387-95.

23. Assunção TS, Ursine PGS. Estudo de fatores associados à adesão ao tratamento não farmacológico em portadores de diabete mellitus assistidos pelo programa Saúde da família, Ventosa, Belo Horizonte. Cien Saude Colet. 2008; 13 (Suppl 2): 2189-2197.

24. Cardozo Gonzales RI, Harter J, Meireles CL, Pinho LB, Lima LM, Reis SP. A descoberta da tuberculose no território: análise qualitativa do trabalho do agente comunitário de saúde. Cienc. enferm. [Internet]. 2015 Ago [citado 18 ene 2017]; XXI (2): 87-97. Disponível em: http://www. scielo.cl/scielo.php?script=sci_arttext $\&$ pid=S0717-95532015000200009\&lng=es

25. Ballarin MLGS, Carvalho FB, Ferigato $\mathrm{SH}$. Os diferentes sentidos do cuidado: considerações sobre a atenção em saúde mental. O Mundo da Saúde São Paulo. 2009; 33(2): 218-224.

26. Ayres JRCM. Cuidado: Trabalho e Interação nas práticas de saúde. Rio de Janeiro: Centro de Estudos e Pesquisas em Saúde Coletiva, Instituto de Medicina Social, Universidade do Estado do Rio de Janei- ro, ABRASCO; 2009. 282 p.

27. Arcêncio RA, Oliveira MF, Cardozo-Gonzales RI, Ruffino-Netto A, Pinto IC, Villa TC. City tuberculosis control coordinators perspectives of patient adherence to DOT in São Paulo State, Brazil. Int J Tuberc Lung Dis. 2008; 12(5): 527-31.

28. Barreira IA. A pesquisa em enfermagem no Brasil e sua posição em agência federal de fomento. Rev Lat Am Enfermagem. 1993; 1(1): 51-57.

29. Caliari JS, Figueiredo RM. Tuberculosis: patients profiles, servisse flowchart, and nurses opinions. Acta Paul Enferm. 2012; 25(1): 43-47.

30. Gomes ALC, Sá LD. As concepções de vínculo e a relação com o controle da tuberculose. Rev Esc Enferm USP. 2009; 43(2): 364-71.

31. Sá LD, Oliveira AAV, Gomes ALC, Nogueira JA , Villa TCS , Collet N. Cuidado ao doente de tuberculose na Estratégia Saúde da Família: percepções de enfermeiras. Rev Esc Enferm USP. 2012; 46(2): 356-63.

32. Coberlline VL. Câncer de mama: encontro solitário com o temor do desconhecido. Rev Gaucha Enferm. 2001; 22(1): 42-68.

33. Bervian PI, Girardon-Perlini NMO. A família (con)vivendo com a mulher/mãe após a mastectomia. Revista Brasileira de Cancerologia. 2006; 52(2): 121-128.

34. Bertolozzi MR, Takahashi RF, Hino P, Litvoc M, França FOS. O controle da tuberculose: um desafio para a saúde pública. Rev Med (São Paulo). 2014; 93(2): 83-9.

35. Mattos RA. A Integralidade na prática (ou sobre a prática da integralidade). Cad. Saude Publica. 2004; 20(5): 14111416. 